REVIEW

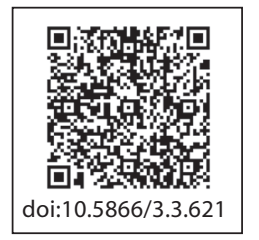

\title{
Host Modulation Therapy - Restriction of Periodontal Destruction
}

\author{
Harinath Reddy $S^{1}$, Satyanarayana $D^{2}$, Vidya Sagar $S^{3}$, Surykanth $M^{4}$
}

Department of Periodontics

Kamineni Institute of Dental Sciences

Narketpally, Nalgonda Dist.

Professor $^{1 \& 2}$

Reader $^{3}$

PG Student ${ }^{4}$

Article Info

Received: April 15, 2011

Review Completed: May, 17, 2011

Accepted: June, 19, 2011

Available Online: October, 2011

(c) NAD, 2011 - All rights reserved

\begin{abstract}
:
Chronic inflammatory periodontal disease is caused by host immune responses to periodontal microorganisms. The past decade has produced remarkable advances in our understanding of host immune responses. New strategies for periodontal disease management have been emerging as more is learned about the role of the host response. Our increasing understanding of inflammation and its resolution has opened the door to the study of new periodontal treatment strategies. The emerging awareness of inflammation and its control in periodontal disease management underscores the importance of exploring inflammatory pathways and mediators, thus setting the stage for the development of new prevention and treatment strategies of a widespread disease.
\end{abstract}

Key words: Host response, HMT, Periodontitis, Periodontal Therapy

\section{INTRODUCTION}

Periodontitis is one of the most common oral diseases and is characterized by gingival inflammation and alveolar bone resorption. ${ }^{1}$ It is estimated that at least $10^{14}$ commensal microbes of various species reside on the surfaces of skin, teeth, dentures, the mucosal epithelial lining of the respiratory, gastrointestinal and urinary tracts, as well as the oral cavity which contains approximately 6 billion microbes representing $500-700$ species. Up to 300 oral bacterial species can be cultured from oral plaque samples. ${ }^{2}$ Hence, it can be considered among the prevalent and important global health problems in terms of quality of life. ${ }^{3}$

The tissue damage and alveolar bone resorption characteristic of the disease are believed to be due to destructive innate host response to pathogenic subgingival biofilm. ${ }^{4}$ Every tooth forms a perforation in the patient's host defence and the body has to work hard to keep the oral microorganisms on the outside. For the maintenance of teeth, the equilibrium between the microbial attack and the host defence is a determining factor. ${ }^{5}$

The underlying biological mechanisms of this response are characterized by the production of hostderived inflammatory mediators including cytokines and lipids by neutrophils, monocytes, lymphocytes and fibroblasts. Acquired and environmental risk factors, such as diabetes mellitus, cigarette smoking and stress, as well as genetically transmitted traits, such as interleukin-1 (IL-1) gene polymorphisms, may accentuate the host inflammatory response to the bacterial challenge and, eventually, the susceptibility to the disease. ${ }^{6}$ Among host proteases degrading extracellular matrix, matrix metalloproteinases (MMPs) seem to be highly related to tissue destruction and remodelling events in periodontal diseases. $^{7}$

Email for correspondence:

harinathsingam@yahoo.com 
New strategies for periodontal disease management have been emerging as more is learned about the role of the host response. Our increasing understanding of inflammation and its resolution has opened the door to the study of new periodontal treatment strategies. ${ }^{8}$

Host modulatory therapy has been proposed as a treatment for periodontal diseases. The use of modulating agents, including inhibition of matrix metalloproteinases (MMPs) with antiproteinases, blocking production of proinflammatory cytokines and prostaglandins with anti-inflammatory drugs, and inhibiting activation of osteoclasts with bonesparing agents, has been postulated to be of therapeutic value as an adjunctive therapy to the management of chronic Periodontitis. ${ }^{9}$ Omega-3 ( $n$ 3) polyunsaturated fatty acids (PUFAs), including docosahexaenoic acid (DHA) and eicosapentaenoic acid (EPA), were shown to have therapeutic antiinflammatory and protective actions in inflammatory diseases including Periodontitis. ${ }^{10}$

The present topic highlights various host modulation therapeutic agents and ongoing development of safe and effective pharmacotherapies that specially target host response mechanism. Introduction of such pharmocotherapies as an adjunct to the traditional periodontal therapies represent a 'new integrated approach' in long-term treatment and management of Periodontitis. The future holds much promise for the host modulation as an important tool not only for the management of Periodontitis, but also for the clinical practice of periodontal medicine.

\section{HOST RESPONSE TO PATHOGENESIS OF PERIODONTITIS}

According to the Merriam-Webster dictionary, the noun "host" has several meanings. Its etymologic origin is from the Latin hostis, which was used to describe a host or guest. In modern English, the word refers to "one that receives or entertains guests socially, non -commercially, or officially". Within the context of immunology, this term specifically refers to the response against parasites. Therefore, "host response in the periodontium" is the defense mechanisms in periodontal tissues against bacterial infections. ${ }^{11}$

The human body is estimated to be composed of more than $10^{14}$ cells, of which only $10 \%$ are mammalian. The majority are the microorganisms that colonize the skin, mouth, digestive, and reproductive tracts. ${ }^{12}$ The resident human microbiota does not merely reside passively at a site, but makes an active contribution to the maintenance of health by promoting the normal development of the physiology of the host (including the immune system), and by excluding exogenous (and often pathogenic) microorganisms (colonization resistance). ${ }^{13}$ In general, the host lives in a relatively stable and harmonious relationship with its resident microbes (termed microbial homoeostasis), and both parties benefit from this symbiosis. ${ }^{14}$

However, if this homoeostasis breaks down, then the dynamic nature of the host-microbe relationship can result in either exogenous microorganisms being able to colonize, or previously minor components of the resident microbiota exploiting new opportunities and increasing in proportion, which in certain circumstances can predispose the site to disease. ${ }^{15}$

\section{The Mouth as a Microbial Habitat}

The mouth provides a warm and moist environment that suits the growth of many microorganisms. The mouth is the only site in the human body that normally provides non-shedding surfaces for microbial colonization; this facilitates the development of thick biofilms, particularly at stagnant sites whereas desquamation ensures that the microbial load is lighter elsewhere. Thus, in this way, the host provides unique opportunities for biofilm formation in the mouth, and a secure haven for microbial persistence. ${ }^{16}$

\section{HOST MODULATORY THERAPY}

Manipulation of the immune response to suppress unwanted reactions is desirable in conditions such as autoimmunity, allergy, or graft 
rejection. It is also required in the case of infectious disease to stimulate the protective processes. Strategies to achieve these goals are collectively referred to as "modulation of host response" and provide a novel concept in treatment. The rationale behind this approach is to aid the host in its fight against infectious agents by supplementing the natural inherent defense mechanisms or to modify its response by changing the course of inflammatory systems. Compared to other weapons against infection, host response modulation potentially has fewer side-effects, is not invasive, and does not require complicated application methods. ${ }^{11}$

\section{DEFINITION AND RATIONALE}

Host modulation therapy (HMT) is a treatment concept that aims to reduce tissue destruction and stabilize or even regenerate the periodontium by modifying or down regulating destructive aspects of the host response and up regulating protective or regenerative responses.

\section{MODULATION OF MATRIX METALLOPROTEINASES}

Matrix metalloproteinases encompass a family of zinc- and calcium-dependent endopeptidases secreted or released by a variety of host cells that function at neutral $\mathrm{p}^{\mathrm{H}}$ and utilize the various constituents of extracellular matrix as their substrates. MMPs can be self-regulated by their own proteolytic inactivation. Some cleavages inactivate MMPs or generate truncated enzyme species resulting in a concomitant change of action. MMPs are also inhibited and cleared by endogenous inhibitors like á2-macroglobulin (á2M) and tissue inhibitors of MMPs (TIMPS), the major plasma inhibitor of MMPs. ${ }^{17}$

\section{TETRACYCLINES IN HOST MODULATION}

The major Antiproteinase used in periodontal treatment is tetracycline (TC). A new approach to non-antibacterial periodontal therapy is the administration of specially prepared low-dose capsules containing as low as $20 \mathrm{mg}$ of doxycycline. Doxycycline is the most potent collagenase inhibitor of commercially available TCs. The CMTs comprise a group of at least 10 (CMTs 1-10) analogues plus some special modified CMTs that differ in their MMP specificity and potency. ${ }^{18}$

\section{MODULATION OF ARACHIDONIC ACID MEDIATORS}

Historically, there have been three major approaches to inhibit $\mathrm{PGE}_{2}$ synthesis. Steroids inhibit PLA2, stabilize lysosomal membranes, and inhibit cellular degranulation, all serving to reduce the availability of free ARA for CO enzymatic activity. Steroids also cause degradation of preexisting mRNAs for IL-1â and TNF-á thereby dampening the secondary $\mathrm{PGE}_{2}$ response. ${ }^{19}$ The second approach is by the use of antioxidants which serve to prevent the oxidation of ARA by molecular oxygen and the subsequent hydrolysis to form $\mathrm{PGE}_{2}$. The third approach is directed towards inhibiting the cyclooxygenase directly. The fact that NSAIDs can suppress alveolar bone resorption suggests that the synthesis of AA metabolites may represent a critical regulatory pathway for potentially blocking periodontal disease progression activity. ${ }^{20}$

A new family of drugs, the cytokine- suppressing anti-inflammatory drugs, has been described of which SKF 86002 is the prototype. These drugs are potent and selective inhibitors of one of the mitogenactivated protein kinase family termed alternatively RK, p38 or cytokine-suppressing anti-inflammatory drugs-binding protein. ${ }^{21}$

\section{POLYUNSATURATED FATTY ACIDS}

Adequate dietary intake of omega-3 (n-3) polyunsaturated fatty acids ( $n-3$ PUFAs) increases tissue concentrations of the types of fatty acids (e.g., eicosapentaenoic acid and docosahexaenoic acid) that downregulate inflammation. ${ }^{22}$ Improved outcomes are attributed to the primary metabolites of omega-3 fish oils, eicosapentaenoic acid (EPA) and docoshexaenoic acid (DHA). ${ }^{23}$

\section{PRORESOLVING LIPID MEDIATORS: POTENTIAL FOR PREVENTION AND TREATMENT OF PERIODONTITIS}

The current therapeutic approach to control inflammation is to remove aetiology. More recently, 
new pathways and processes underlying resolution of inflammation have been discovered stimulating increased interest in proresolving lipid mediators of inflammation. The Proresolving molecules include lipoxins that are produced from the metabolism of endogenous arachidonic acid (AA) and resolvins that are derived from dietary omega-3 polyunsaturated fatty acids ( $n-3$ PUFA). Resolvins and protectins are two new families of compounds identified in the resolution of inflammation. ${ }^{24}$

\section{MODULATION OF HOST CELL RECEPTORS: CYTOKINES}

Inflammatory cytokines are thought to trigger periodontal tissue destruction. In addition to being regulated by anti-inflammatory mediators, their activity is under the control of suppressors of cytokine signaling (SOCS), which down-regulate the signal transduction as part of an inhibitory feedback loop. The increased expression of SOCS-1, -2 and -3 mRNA in diseased periodontal tissues is believed to be involved in the down-regulation of inflammatory cytokine and Toll-like receptor signaling. ${ }^{25}$

The immunization of non-human primates in ligature-induced periodontitis models with $P$. gingivalis or a $P$. gingivalis virulence factor called cysteine protease, has demonstrated partial reductions in alveolar bone loss. ${ }^{26}$

\section{Pentoxifylline}

Pentoxifylline (PTX), a methylxanthine derivative, specially blocks the synthesis of TNF-á, among other cytokines, by inhibiting gene transcription, thereby reducing the accumulation of TNF-á mRNA. The protective effect of PTX could be explained by its capacity to inhibit the production of inflammatory cytokines or to stimulate anti-inflammatory cytokine production. ${ }^{27}$

\section{MODULATION OF BONE REMODELLING}

Factors regulating osteoblast and osteoclast activity have become important targets for developing pharmacological and clinical strategies to modulate the rate of bone formation and resorption. The identification of the interaction between RANKL and OPG has recently received attention in periodontal research.

\section{Bisphosphonates}

Bisphosphonates are 'bone-sparing' agents used in the management of various diseases with bone resorption. These compounds inhibit osteoclastic activity by blocking acidification by local release and represent a class of chemical structures related to pyrophosphate. ${ }^{28}$ In a recent study by Tipton et al. ${ }^{29}$ Human gingival fibroblasts were derived from explants obtained from healthy individuals with noninflamed gingiva. The effects of alendronate and pamidronate on the constitutive production, or the lipopolysaccharide (LPS)- or IL-1â-stimulated production, of IL-6, RANKL and OPG by human gingival fibroblasts was determined and concluded that LPS and BPs were not cytotoxic, BPs decreased the production of LPS- or IL-1â-stimulated RANKL and decreased constitutive, LPS-stimulated and IL-1âstimulated RANKL/OPG ratios. In addition, recent media reports announced that cases of jaw Osteonecrosis occurred in a trial of zoledronic acid or denosumab (Amgen, Thousand Oaks, CA, USA) for bone metastasis. Denosumab is a fully human monoclonal antibody against receptor activator of nuclear factor- ${ }^{\circ}$ ligand (RANKL). ${ }^{30}$

\section{PROBIOTICS}

Chronic periodontitis could benefit from orally administered probiotics. The presence of periodontal pathogens could be regulated by means of antagonistic interactions. A decrease in gum bleeding and reduced gingivitis has been observed with the application of Lactobacillus reuteri. Probiotic strains included in periodontal dressings at optimal concentration of $108 \mathrm{CFU} \mathrm{ml}$ were shown to diminish the number of most frequently isolated periodontal pathogens: Bacteroides sp., Actinomyces sp. and S. intermedius, and also C. albicans. ${ }^{31}$

\section{WHICH SUBJECTS WOULD PARTICULARLY BENEFIT FROM HOST MODULATION THERAPY (HMT)?}


Conventional periodontal therapy is effective in the management of most cases of periodontitis. However, there are sub-optimally responding subjects and identifiable high-risk groups where adjunctive HMT may have utility. Further research is required to test the efficacy, cost effectiveness and cost utility of HMT in these groups. It will be necessary in future to test these added benefit assumptions in clinical trials of adjunctive host modulation in highrisk groups. ${ }^{32}$

\section{POSSIBLE STRATEGIES IN THE FUTURE}

\section{Anti-Porphyromonas Gingivalis Egg Yolk Antibody}

Pretreatment of gingipains with IgY-GP was associated with strong inhibition of cell detachment, antibody against gingipains [immunoglobulin $Y$ (IgY)-GP] on gingipain activity in vitro. The findings suggest that IgY GP may be an effective immunotherapeutic agent in the treatment of periodontitis. $^{33}$

\section{CONCLUSION}

The adjunctive use of host modulatory therapy can enhance therapeutic responses, slow the progression of the disease, and allow for more predictable management of patients, particularly in those patients at increased risk caused by factors beyond the reach of conventional therapeutic approaches. The field of "perioceutics", or the use of pharmacological agents specifically developed to better manage periodontitis, is emerging to aid in the management of these susceptible patients who develop periodontal disease. Host modulatory therapy, which can be used to bring down excessive levels of enzymes, cytokines, and prostanoids as well as modulate osteoclast function, is the key to addressing many of these risk factors that have adverse effects on the host response. ${ }^{34}$

Although, innumerable therapies are beginning to surface, a handful of these have true potential in clinical practice. We have ahead of us a Herculean task of identifying those strategies with the promise of clinical applicability and developing them to better suit all patients.

\section{REFERENCES}

1. Savage, A., Eaton, K. A., Moles, D. R. \& Needleman, I. A systematic review of definitions of Periodontitis and methods that have been used to identify this disease.J Clin Periodontol 2009; 36, 458-467.

2. Jin L. An update on innate defense molecules of human gingiva. Periodontology 2000 2011; 56, 125-142.

3. Buduneli N, Kinane D F. Host-derived diagnostic markers related to soft tissue destruction and bone degradation in periodontitis.J Clin Periodontol 2011;38 (Suppl. 11), 85-105.

4. Brain W. Bainbridge, Stephen R. Coats, and Richard P. Darveau. Porphyromonas gingivalis Lipopolysaccharide Displays Functionally Diverse Interactions with the Innate Host Defense System. Ann Periodontol 2002; 7, 29-37.

5. Listgarten M. A. A perspective on periodontal diagnosis. J Clin Periodontol 1986; 13,175-181.

6. Salvi GE, Lang NP. Host response modulation in the management of periodontal diseases. J Clin Periodontol 2005; 32 (Suppl. 6), 108-129.

7. Dahan M. Nawrocki B, Elkaim R, Soell M, Bolcato-Bellemin $A-L$, Birembaut P,Tenenbaum $H$. Expression of matrix metalloproteinases in healthy and diseased human gingiva. J Clin Periodontol 2001; 28, 128-136.

8. Bhatavadekar NB, Williams RC. Commentary: New directions in host modulation for the management of periodontal diseases. J Clin Periodontol 2009 36, 124-126.

9. Michael S. Reddy, Nico C. Geurs, and, and John C. Gunsolley. Periodontal Host Modulation with Antiproteinase, AntiInflammatory, and Bone-Sparing Agents. A Systematic Review. Ann Periodontol 2003; 74, 12-37.

10. Hesham El-Sharkawy, Nayer Aboelsaad, Mohamed Eliwa, Mahmoud Darweesh, Mohammad Alshahat, Alpdogan Kantarci, Hatice Hasturk, and Thomas E. Van Dyke. Adjunctive Treatment of Chronic Periodontitis with Daily Dietary Supplementation with Omega-3 Fatty Acids and Low-Dose Aspirin. J Periodontol 2010; 81, 1635-1643.

11. Kantarci A, Hatice Hasturk \& Thomas E. van Dyke. Hostmediated resolution of inflammation in periodontal diseases. Periodontology 2000 2006; 40, 144-163.

12. Wilson, M. Microbial Inhabitants of Humans. Their Ecology and Role in Health and Disease. 2005; Cambridge: Cambridge University Press.

13. Wilks, M. Bacteria and early human development. Early Human Development 2007; 83, 165-170.

14. Marsh, P.D. Host defenses and microbial homeostasis: Role of microbial interactions. J Dent Res 1989; 68, 1567-1575.

15. Marsh PD, Devine DA. How is the development of dental biofilms influenced by the host? J Clin Periodontol 2011; 38 (Suppl. 11), 28-35. 
16. Marsh, P.D., Moter, A.\& Devine, D.A. Dental plaque biofilms: communities, conflict and control. Periodontology 2000 $2011 ; 55,16-35$.

17. Ryan \& Lorne M. Golub. Modulation of matrix metalloproteinase activities in periodontitis as a treatment strategy. Periodontology 2000 2000; 24, 226-238.

18. Golub LM, Lee HM, Ryan ME et al. Tetracyclines inhibit connective tissue breakdown by multiple non-anti microbial actions. Adv Dent Res 1998; 12, 12-26.

19. McGuire J, Langdon R, Birchall N, Kupper T. Interleukin -1 alpha mRNA induced by cyclohexamide, $\mathrm{PMA}$, and retinoic acid is reduced by dexamethasone in PAM-212 keratinocytes. Ann N Y Acad sci 1989; 548, 283-290.

20. Morton RS, Dongari-Bagtzoglou Al. Cyclooxygenase-2 is upregulated in inflamed gingival tissues. J Periodontol $2001 ; 2,461-469$.

21. Erica Gemmell, Roderick I. Marshall \& Gregory. J Seymour. Cytokines and prostaglandins in immune homeostasis and tissue destruction in periodontal disease. Periodontology 2000 1997; 14, 112-143.

22. Ziboh, V. A. Nutritional modulation of inflammation by polyunsaturated fatty acids/eicosanoids. In: Gershwin, M. E., German, B. J. \& Keen, C. L. (eds). Nutrition and Immunology: Principles and Practice, 2000; pp. 157-167. Totowa, NJ: Humana.

23. Van der Velden U, Kuzmanova D, Chapple ILC. Micronutritional approaches to periodontal therapy. J Clin Periodontol 2011; 38 (Suppl. 11), 142-158.

24. Serhan CN, Hong S, Gronert K, et al. Resolvins: A family of bioactive products of omega-3 fatty acid transformation circuits initiated by aspirin treatment those counter proinflammation signals.J Exp Med 2002; 196, 1025-1037.

25. Garlet GP, Cardoso CR, Campanelli AP, Martins W Jr, Silva JS. Expression of suppressors of cytokine signaling in diseased periodontal tissues: a stop signal for disease progression? J Periodont Res 2006; 41, 580-584.

26. Ebersole JL, Brunsvold M, Steffensen B, et al. Effects of immunization with Porphyromonas gingivalis and Prevotella intermedia on progression of ligature-induced periodontitis in the nonhuman primate Macaca fascicularis. Infection Immunity 1991; 59, 3351-3359.

27. Lima V, Brito GAC, Cunha FQ, Rebouc, as CG, Falcao BAA, Augusto RF, Souza MLP, Leitao BT, Ribeiro RA. Effects of the tumour necrosis factor-á inhibitors Pentoxifylline and thalidomide in short-term experimental oral mucositis in hamsters. Eur J Oral Sci 2005; 113, 210-217.

28. Rogers MJ, Gordon S, Benford HL, Coxon FP, Luckman SP, Monkkonen J, Frith JC. Cellular and molecular mechanisms of action of bisphosphonates. Cancer 2000; 88, 2961-2978.

29. Tipton DA, Seshul BA, Dabbous MKh. Effect of bisphosphonates on human gingival fibroblast production of mediators of osteoclastogenesis: RANKL, osteoprotegerin and interleukin-6.J Periodont Res 2011; 46, 39-47.

30. Borromeo GL, CE Tsao, IB Darby, PR Ebeling.. A review of the clinical implications of bisphosphonates in dentistry. Aust Dent J 2011; 56, 2-9.

31. Meurman JH, I Stamatova. Probiotics: contributions to oral health. Oral Diseases 2007; 13, 443-451.

32. Kinane DF, Attstrom R. Advances in the pathogenesis of periodontitis consensus report of the fifth European workshop in Periodontology. J Clin Periodontol 2005; 32 (Suppl. 6), 130-131.

33. Yokoyama K, Sugano N, Rahman AKMS, Oshikawa M, Ito K Activity of anti- Porphyromonas gingivalis egg yolk antibody against gingipains in vitro. Oral Microbiol Immunol 2007; 22, 352-355

34. Maria Emanuel Ryan. Clinical applications for host modulatory therapy. Compend Contin Educ Dent 2002; 23 (11A), 1071-6.

\section{Gain quick access to our journal online View our journal at

\author{
wWW.nacd.in
}

